wave functions so that the interference effect is sensitive to weak magnetic fields unlike the interaction effect. This very different magnetic field dependence enables the two effects to be separated experimentally. Heavy impurities lead to a large spinorbit coupling in the scattering process and a rotation of the spin of the electrons. This in turn changes the sign of the interference effect. Thouless had shown that inelastic scattering events lead to a total loss of phase memory and that a study of the temperature dependence of the interference effect determines the inelastic scattering rate. All these features were demonstrated in the series of experiments that Bergmann presented.

Bruynseraede introduced work done by the Leuven group in collaboration with G. Deutscher (Tel-Aviv) which also showed the weak localization effect and in addition studied the interplay of this effect with superconducting fluctuations in Al films that are superconducting at low temperature. This interplay had been discussed theoretically recently by Larkin and the data presented by Bruynseraede clearly verified the theory.

In three dimensions there is a real metalinsulator transition, but over the years there has been no unanimity on whether this transition is continuous or discontinuous. For example, Mott has argued for a minimum value for the conductivity of a metal which requires a discontinuous jump in the conductivity extrapolated to $T=0 \mathrm{~K}$. On the other hand, the scaling theory of the "gang of four" predicts a continuous transition at $T=0 \mathrm{~K}$ with critical exponents for the divergence of the resistivity. Recently the classic system of a doped semiconductor, $\mathrm{Si}: \mathrm{P}$, has been investigated in a careful series of experiments by a group at Bell Labs. IG.A. Thomas, M. Paalanen, T.F. Rosenbaum and R.N. Bhatt). They worked at much lower temperatures, down to the millikelvin range, and they performed a very elegant series of experiments using external stress to sweep their samples through the metal-insulator transition. Their results were presented by Paalanen at the meeting, who explained that a continuous transition with no sign of the minimum metallic conductivity had been found, but the critical exponents do not agree with those predicted by the single-electron scaling theory, suggesting an important role for electron-electron effects.

While the theory of the weak localization regime in two dimensions is by now well established, the theory of the metal-insulator transition in three dimensions is still being actively studied. One can use the weak localization theory to give the first terms in the scaling equations but a treatment of the actual metal-insulator transition, and a determination of the scaling behavior including both disorder and interaction ef- fects, require higher order terms and the solution of a complicated series of scaling equations. This point was stressed by $C$. Castellani (Rome) who presented the latest theoretical work of this group (C. Castellani, C. DiCastro, G. Forgacs and E. Tahet). The problem is also being studied by Grest and P.A. Lee who recently published results of a scaling approach which gave good agreement with the Bell group's experiments. However Castellani showed that terms neglected in the GrestLee approach may influence the results considerably. The progress in this theory by these groups has been impressive but the final word is not yet in.

An alternative approach by the group at Munich (A. Gold and W. Götze), presented by Gold, uses a generalized self-consistent current relaxation theory. Their theory has the advantage that it can be applied to give a general phase diagram of the metal-insulator transition.

The special case of one dimension was discussed by $W$. Apel, presenting work done with the author in Zürich. He covered the case of materials which are one-dimen- sional as regards the electronic interaction but do not have a Peierls transition. He showed that the divergences introduced by interactions in $\mathrm{d}=1$ modify the asymptotic behaviour in the scaling theory of "the gang of four" and lead to a transition between a localized insulating state and a perfectly conducting metallic state. $\mathrm{He}$ also gave a more intuitive picture in terms of a crossover between quantum and classical transport as the ratio of elastic and inelastic scattering lengths change.

\section{Conclusion}

Progress made in the past few years in this field has been remarkable. Not only have spectacular new and unsuspected effects, such as the absence of true metallic conduction in two dimensions been discovered, but also a much better understanding of the metal-insulator transition has been obtained. The goal of a complete understanding, which has eluded us for more than 40 years, is in sight. The talks at the meeting conveyed this progress but gave just a selection of the work underway in many groups in all parts of the world.

\title{
Itinerant Electron Magnetism
}

\section{E. P. Wohlfarth, London \\ (Imperial College)}

Itinerant electron magnetism as a subject had its birth in the 1930's and, after a lengthy period of relative neglect, it experienced a renaissance beginning about 20 years ago. At the present time it has reached an exciting stage of development to which there is no rapid end in sight. Contributing to the evolution of the subject, concerned as it is with the magnetic properties of metals and alloys, are: theoretical sophistication, advanced computations and advances in experimental techniques. All three of these facets appeared in an exciting way at a recent Workshop on 3d metallic magnetism, held in Grenoble on 25-26 March, 1983, and at the 3rd General Conference of the Condensed Matter Division in Lausanne. Itinerancy between these two towns was provided by a bus!

The simplest model of metallic magnetism is that of Stoner to which Mott and Slater had made essential contributions. The itinerant electrons are regarded as being distributed in single particle energy levels with characteristically complicated density of states curves $N(\varepsilon)$. The manybody electron interactions leading to enhanced Pauli paramagnetism or, beyond a certain strength, to itinerant ferromagnetism, are described in this approach by a mean-field approximation. The essential contributions to the mean field energy $I$ come from intra-atomic Coulomb interactions and, since the resulting Hartree-Fock approximation is inapplicable, from correlations between antiparallel spins. This approach has been found in the last few years to be a relatively good description of metallic magnetism at $0 \mathrm{~K}$, using for this confirmation a theoretical approach called the density functional formalism. Even so, this approach is not always satisfactory: It overestimates the exchange splitting between opposite spins in nickel by a factor of about 2 when compared to the value obtained experimentally using photoemission techniques. However, the greatest controversy at present concerns the description of metallic magnetism at finite temperatures, up to and beyond the Curie point $T_{\mathrm{c}}$. In the Stoner theory this was based on the straightforward use of Fermi statistics, characterized by the usual distribution function $f(\varepsilon, T)$. A particularly heated controversy concerns the resulting value of $T_{c^{\prime}}$, given by:

$$
/ \int_{0}^{\infty} N(\varepsilon)|\partial f / \partial \varepsilon|_{T=T_{c}} d \varepsilon=1 .
$$

A folklore has developed spontaneously that this $T_{c}$ is larger, by factors of about 5 , than the measured values for iron, cobalt and nickel. However, as was pointed out during the discussion at Grenoble, a value of $I$, the mean field energy, in agreement with the photoemission data rather than that calculated by the density functional formalism, gives reasonable values of $T_{c}$ for 
nickel. For iron, the situation is completely different. Since the number of magnetic "particles" (d-band holes) is here much larger than for nickel, the intra-atomic exchange which is not included in the mean field energy, basic to the Stoner model, must be extraordinarily important. This effect leads to Hund's rule in atoms and is central to the ferromagnetism of iron.

For this metal the greatest controversy appeared to be the behaviour above $T_{c^{\prime}}$ in the following sense: The Stoner model was improved early in its history by calculating, on the same basis, the collective excitations of electron-hole pairs called spin waves or magnons. The spin wave energy at small wave vectors $q$ is given by

$$
\hbar \omega_{q}=D q^{2} \text {, }
$$

with $D$ the so-called spin-wave stiffness. These excitations, observable by neutron scattering, should vanish above $T_{c}$, but this is apparently not the case, at least for iron (for nickel there are also "features" in the $\omega, q$ plane above $T_{c}$ but it is again not clear in what sense these are spin waves). As a result, a model of extended regions of magnetic order above $T_{c}$ has been proposed such that spin waves can be supported therein. Perhaps the most heated debate in Grenoble, also reflected at the Lausanne meeting, concerned the reality of these regions. As was pointed out, their existence would lead to extremely large local magnetic moments above $T_{c}$ which are simply not observed by static susceptibility measurements (Curie-Weiss law). An alternative model was described which for iron involves a strong localization of magnetic moments due to the Hund's rule coupling already mentioned. The static susceptibility now corresponds to a small number of spins, in agreement with observation. The corresponding dynamical susceptibility was shown to be in good agreement with the neutron data at small wave vectors $q$.

However, so potent was the influence of the model of large regions of order that it also affected the interpretation of the photoemission data for nickel, as reported in Grenoble. If this model is used, these data have to be interpreted with a 3-peak fit and the outermost peaks are then found to stay apart above $T$, in disagreement with the Stoner model. Other photoemission data on Ni were, however, interpreted with a 2-peak fit such that now the two peaks coalesce at high temperatures, in closer agreement with this model which thus has a good chance of being more reasonable for $\mathrm{Ni}$ than had been supposed, apparently due to the weak Hund's rule coupling compared to its strength in $\mathrm{Fe}$.

The overwhelming interest in iron and nickel did not prevent other theoretical and experimental concepts and other materials from being discussed in considerable depth. Whereas spin waves are important elementary excitations in ferromagnetic metals at low temperatures, other similar spin fluctuations should occur for materials which are only weakly itinerant ferromagnets or are indeed strongly enhanced paramagnets. The latter situation arises in palladium and there the influence of "paramagnons" on the magnetic and other properties is still debatable. What, for example, is the effect of the complicated structure of the $3 \mathrm{~d}$ energy bands? Very weakly itinerant ferromagnets are now known to be very numerous. A textbook example is the material $\mathrm{ZrZn}_{2}$ which is ferromagnetic, with a Curie point $T_{\text {c }}$ at about $25 \mathrm{~K}$, due to a sharp peak in its density of states curve. Highly sophisticated finite temperature band structure investigations using the de Haas-van Alphen effect were reported as showing a dependence of the exchange splitting between opposite spins in agreement with the Stoner model. Other materials of this type were discussed with the following question in mind: What is the relative importance of the amplitudes of the spin fluctuations compared to those of concentration fluctuations or other structural effects due to the complicated metallurgy? A systematic study may be based on high magnetic field data (curvature of Arrott plots giving $M^{2}$ vs. $H / M$, where $M$ is the magnetization in a field $H$ ) and on magnetovolume effects (dependence of $d T_{c} / d P$ on $T_{c}$ for a series of alloys with varying Curie temperatures and under a pressure $P$ ). This study, backed by the Landau-Ginzburg theory of phase transitions involving a spatially varying magnetization, seems to highlight clearly the importance of structural heterogeneities in magnetic alloys.

Magnetovolume effects are now realized to be of central importance in comparing models of itinerant electron magnetism. Apart from the pressure dependence of $T_{c}$ there is also that of the saturation magnetization (how do the respective logarithmic derivatives differ?), as well as volume and forced magnetostrictions, thermal expansion, linear magnetostriction and magnetic contributions to the elastic constants. A wide range of weakly itinerant alloys has been investigated as to their magnetovolume effects. These studies are related to the question of the persistence of magnetic moments above $T$ as engendered by spin fluctuation effects. Unfortunately, it is notoriously difficult to disentangle the various magnetic and nonmagnetic thermal expansion contributions above and below $T_{c}$ and this approach is as yet inconclusive. The same applies to the data for pure iron and nickel.

There is additionally great interest in the itinerant electron magnetism of rare earth (including $Y$ !) and actinide containing compounds, and this was reflected in talks at Grenoble and Lausanne. The phenomena observed in the range of the onset of $3 d$ magnetism show a rich variety of behaviour patterns which encompass several recent

\section{SUNY Stony Brook}

Post-doctoral position is available for an experimental physicist with a background in surface physics.

Experience in any or all of the following fields is desirable: ultra-high vacuum, LEED, AES, UPS, ISS.

Research in surface crystallography will involve correlated experiments at the storage ring (NSLS) and in the laboratory.

Please send résumé, list of publications and references to:

\section{Dr. F. Jona,}

Department of Materials

Science and Engineering, SUNY Stony Brook,

Stony Brook,

N.Y. 11794, U.S.A.

AK 598 B

physical ideas: Strong paramagnetism was observed in $\mathrm{YNi}_{5}, \mathrm{CeNi}_{5}, \mathrm{YCO}_{2}$, the latter having many similarities with $\mathrm{Pd}$ and thus perhaps liable to paramagnon effects. In other compounds, $\mathrm{ThCo}_{5}, \mathrm{Ce}(\mathrm{Co}, \mathrm{Ni})_{5}$, $\mathrm{Y}_{2} \mathrm{Ni}_{17}, \mathrm{RCo}_{2}(\mathrm{R}=\mathrm{Dy}, \mathrm{Ho}, \mathrm{Er})$, band structure engendered first order or metamagnetic transitions have been observed in high magnetic fields. For $\mathrm{Y}_{2} \mathrm{Ni}_{7}$ an effect called spontaneous thermal magnetization (equivalent to two critical temperatures) was observed and also related to band structure effects. Finally, $\mathrm{YNi}_{3}$ exhibits very weak itinerant ferromagnetism of about the same high quality as $\mathrm{ZrZn}$.

Among actinide compounds, particular interest attaches to those of uranium. Thus $\mathrm{UFe}{ }_{2}$ (and indeed $\mathrm{U}\left(\mathrm{Fe}_{1-\mathrm{x}} \mathrm{Co}_{\mathrm{x}}\right)_{2}, \mathrm{x} \leq 0.3$ ) exhibits magnetic and magnetovolume effects characteristic of itinerant ferromagnetism, as does the compound UPt, although with reservations. The paramagnetic Laves phase compound $\mathrm{UAl}_{2}$ has specific heat anomalies ascribed to spinfluctuation effects. In all these materials, it is important to note that the $5 \mathrm{f}$ bands have a considerably greater width than the $4 f$ levels in the rare earths, so that itinerant effects recalling those of the transition metals and alloys are rather natural.

In view of all this richness, it seems rather carping to conclude by regretting the virtual absence of any reference to the itinerant magnetism in amorphous alloys. 